\title{
Skyrmions in a frustrated model of multiferroic superlattices
}

\author{
I. F. Sharafullin ${ }^{\dagger}$, N. M. Nugaeva, M. Kh. Kharrasov \\ †SharafullinIF@yandex.ru
}

Bashkir State University, 32 Zaki Validi St., Ufa, 450076, Russia

In this work we study phase transitions and surface properties of multiferroic superlattice by Monte-Carlo simulation. We consider a multilayer film of a multiferroic consisting of $L_{z}^{m}$ ferromagnetic layers and $L_{z}^{f}$ ferroelectric layers sandwiched in the $z$-direction. Each $x y$ plane has dimension $L \times L$. The magnetic film we consider as a film with a body-centered cubic lattice, the ferroelectric film as a film with body-centered cubic lattice. We have studied a new model for the interface coupling between a ferromagnetic film and a ferroelectric film in a superlattice of multiferroic. This interaction has the form of a Dzyaloshinskii-Moriya (DM) interaction between the order parameters of ferroelectric films and the spins of ferromagnetic layers at the interface. We have taken into account the frustration due to the NNN interactions in both magnetic and ferroelectric layers. The ground state shows uniform non collinear spin configurations in zero field and skyrmions in an applied magnetic field. Monte Carlo simulation has been used to study the phase transition occurring in the superlattice with and without applied field. Skyrmions have been shown to be stable at finite temperatures and up to finite values of the NNN exchange interactions. We have also shown that the nature of the phase transition can be of second or first order, depending on the value of magnetoelectric interaction. As expected, the magnetic frustration enhances creation of skyrmions. The existence of skyrmions confined at the ferromagnetic-ferroelectric interface is very interesting. For MC simulations, we use the Metropolis algorithm for a system with linear dimensions $L \times L \times L_{z}$.

Keywords: superlattice, skyrmions, Monte-Carlo modeling, frustrated models, magnetoelectric interaction.

\section{Introduction}

The past decade was characterized by a significant increase in activity in the field of materials research, in which the interrelation of magnetic and electrical properties is manifested. Recently, magnetic/ferroelectric superlattices attract much of attention as magneto-electric (ME) materials. There are intrinsic magneto-electric effects due to spin-orbit interaction [1] as well as spin charge-orbital coupling [2]. Surface ME effects appears due to the charge and spin accumulation [3-5]. The enhancement of magnetoelectric effect due to phase separation was shown in [6]. In non collinear structures, the microscopic mechanism of the coupling of polarization and the relative orientation of the magnetization vectors is based on the interaction of Dzyaloshinskii-Moriya $[7,8]$. In some micromagnetic structures all ligands are shifted in one direction, which leads to the appearance of macroscopic electrical polarization. In the case of magnetically ordered matter, the contribution of the Dzyaloshinskii-Moriya interaction to the free energy can be represented as Lifshitz antisymmetric invariants containing spatial derivatives of the magnetization vector. In analogy, the vortex magnetic configuration can be stable via Skyrme mechanism [9]. For a long time skyrmions have been the subject only of theoretical studies. In particular, it was shown that such structures can exist in magnetic and ferroelectric materials $[10,11]$. The superstructures naturally lead to the interaction of skyrmions on different interfaces, which has unique dynamics compared to the interaction of the same-interface skyrmions. Information flow in nextgeneration spintronic devices could be associated with metastable isolated skyrmions guided along magnetic strips [13 - 15]. In [16] have been studied effects of DzyaloshinskiiMoriya (DM) magnetoelectric coupling between ferroelectric and magnetic layers in a superlattice formed by alternate unfrustrated magnetic and ferroelectric films. Using the Green's function method, it was shown that the spin waves excited in a monolayer and also in a bilayer sandwiched between ferroelectric films, in zero field, the DM interaction strongly affects the long-wavelength SW mode. H. T. Diep et al. [17] have studied a crystal of skyrmions generated on a square lattice using a ferromagnetic exchange interaction and a Dzyaloshinskii-Moriya interaction between nearestneighbors under an external magnetic field. They have shown that the skyrmion crystal has a hexagonal structure which is shown to be stable up to a temperature $T_{c}$ where a transition to the paramagnetic phase occurs and the dynamics of the skyrmions at $T<T_{c}$ follows a stretched exponential law. We consider in this paper a superlattice composed of alternate magnetic films and ferroelectric films. The aim of this chapter is to propose a new model for the coupling between the frustrated magnetic film and the frustrated ferroelectric film by introducing a DM-like interaction.

\section{Model and ground state of skyrmion cristall}

Consider a superlattice composed of alternate magnetic and ferroelectric films. Both have the structure of body-centered 
cubic lattice of the same lattice constant, for simplicity. The Hamiltonian of this multiferroic superlattice is expressed as:

$$
H=H_{m}+H_{f}+H_{m f}
$$

where $H_{m}$ and $H_{f}$ are the Hamiltonians of the magnetic and ferroelectric subsystems, respectively, $H_{m f}$ denotes Hamiltonian of magnetoelectric interaction at the interface of two adjacent films. We are interested in the frustrated regime. Therefore we describe the Hamiltonian of the magnetic film with the Heisenberg spin model on a bodycentered cubic lattice as follows:

$$
H_{m}=-\sum_{i, j} I_{i j}^{m} \vec{S}_{i} \cdot \vec{S}_{j}-\sum_{i, k} I_{i k}^{m} \vec{S}_{i} \cdot \vec{S}_{k}-\sum_{i} \vec{H} \cdot \vec{S}_{i}
$$

where $\vec{S}_{i}$ is the spin on the $i$-th site, $\vec{H}$ the external magnetic field, $J_{i j}^{m}$ the magnetic interaction between two spins at $i$ and $j$ sites. We shall take into account both the nearest neighbors (NN) interaction, denoted by $J^{m}$, and the next-nearest neighbor (NNN) interaction denoted by $I^{m}$. We consider ferromagnetic interaction $J^{m}>0$ to be the same everywhere in the magnetic film. To introduce the frustration we shall consider antiferromagnetic interaction $I_{\vec{m}}^{m}$, also the same everywhere. The external magnetic field $\vec{H}$ is applied along the $z$-axis which is perpendicular to the plane of the layers.

For the ferroelectric film, we suppose for simplicity that electric polarizations are vectors of magnitude $|\vec{P}|=1$, pointing in the $\pm z$ direction. The Hamiltonian is given by

$$
H_{f}=-\sum_{i, j} J_{i j}^{f} \vec{P}_{i} \cdot \vec{P}_{j}-\sum_{i, k} I_{i k}^{f} \vec{P}_{i} \cdot \vec{P}_{k}
$$

where $\vec{P}_{i}$ is the polarization on the $i$-th lattice site, $J_{i j}^{f}$ the interaction parameter between polarizations at sites $i$ and $j$. Similar to the magnetic subsystem we will take the same ferroelectric interaction $J_{i i}^{f}=J^{f}$ for all NN pairs of polarizations, and $I_{i k}^{f}=I^{f}<0$ for NNN pairs.

For the magnetoelectric interaction at the interface, we choose the interface Hamiltonian following Ref. [16]:

$$
H_{m f}=\sum_{i, j, k} J^{m f} e_{i, j} \vec{P}_{k} \cdot\left[\vec{S}_{i} \times \vec{S}_{j}\right]
$$

where $\vec{P}_{k}$ is the polarization at the site $k$ of the ferroelectric interface layer, while $\vec{S}_{i}$ and $\vec{S}_{j}$ belong to the interface magnetic layer. In this expression $J^{m f} e_{i, j} \vec{P}_{k}$ plays the role of the DM vector perpendicular to the $x y$ plane, given by Eq. (4). When summing the neighboring pairs $(i, j)$, attention should be paid on the signs of $e_{i j}=-e_{j i}=1$ and $\left[\vec{S}_{i} \times \vec{S}_{j}\right]$. We note that the vector product $\left[\vec{S}_{i} \times \vec{S}_{j}\right]$ changes its sign with the permutation of $i$ and $j$. But the whole Hamiltonian of magnetoelectric interaction (Eq. (4)) should be invariant under permutations of these indices. This explains why we need the coefficient $e_{i, j}=-e_{j, i}=1$ present in Eq. (4).

Since $\vec{P}_{k}$ is in the $z$ direction - the DM vector is in the $z$ direction, in the absence of an applied field the spins in the magnetic layers will lie in the $x y$ plane to minimize the interface interaction energy, according to Eq. (4). The magnetoelectric interaction $J^{m f}$ favors a canted (non collinear) spin structure. It competes with the exchange interactions $J^{m}$ and $I^{m}$ of Eq. (2) which favor collinear (ferro and antiferro) spin configurations. In nanofilms of superlattices the magnetoelectric interaction is crucial for the creation of non-collinear long-range spin order. DM interaction has been identified as a key ingredient in the creation, stabilization of skyrmions and chiral domain walls $[18,19,16,22]$.

In the case when the magnetic film has a frustration and a thickness, the angle between NN spins in each magnetic layer is different from that of the neighboring layer. This makes the determination of the angles analytically difficult. It is more convenient to use the numerical minimization method called "steepest descent method" to obtain the ground state (GS) spin configuration. This method consists in minimizing the energy of each spin by aligning it parallel to the local field acting on it from its neighbors. We use a sample size $N \times N \times L$. We apply a magnetic field perpendicular to the $x y$ plane. For most calculations, we select $N=60$ and $L=8$ using the periodic boundary conditions in the $x y$ plane. For simplicity, when we investigate the effect of the exchange couplings on the magnetic and ferroelectric properties, we take the same thickness for the upper and lower layers $L_{m}=L_{f}=4=L / 2$. All the results are obtained with exchange parameters between $\mathrm{NN}$ spins and NN polarizations are taken as $J_{m}=J_{f}=1$ for different values of the interaction parameters between NNN spins and NNN polarizations $\left(I^{m}, I^{f}\right)$ and $J^{m f}$. We note that the steepest descent method calculates the real GS down to the value $J^{m f}=-1.15$. For values lower than this, the angle between two spins at $i$ and $j$ sites tends to $\pi / 2$ so that all magnetic and ferroelectric exchange terms will be close to zero, the minimum total energy corresponds just to the DM energy.

The spin configuration in the case where $H=0$ is shown in Fig. 1 for the interface magnetic layer with frustration of the magnetic and ferroelectric layers. We observe here for the case without frustration the GS spin configurations have periodic structures without mixed domains (not shown) and in second case $\left(I^{m}=I^{f}=-0.3\right)$ a stripe phase with long islands and domain walls. The inside magnetic layers have the same texture.

In the present system, there is a competition between the applied field which wants to align the spins along the $z$ direction, and the DM interaction which wants the spins to be perpendicular which each other in the $x y$ plane. As a consequence, spins find a compromise which is the structure of skyrmions as shown below. Fig. 2 shows the 3D ground state configuration for $J^{m f}=-1.22$ and $J^{m}=J^{f}=1.0, I^{m}=I^{f}=0$ for first (surface) magnetic layer, with external magnetic layer $H=0.15$.

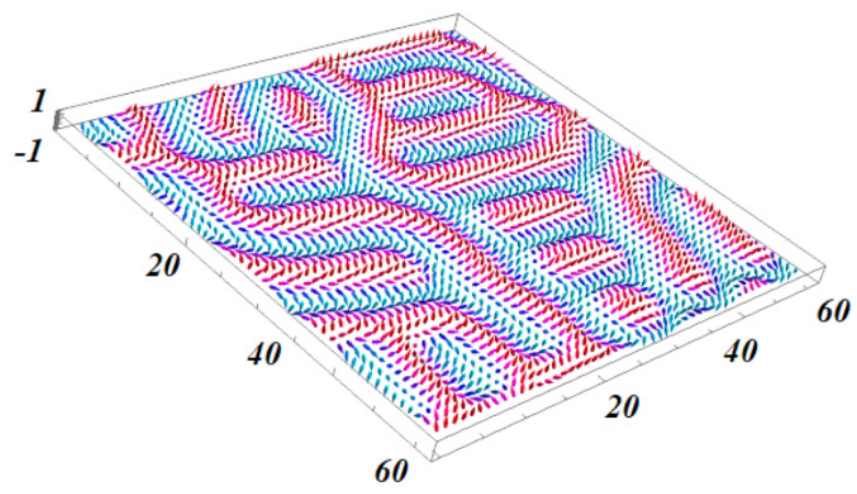

Fig. 1. (Color online) $3 \mathrm{D}$ view of the GS configuration of the surface magnetic layer for $J^{m f}=-1.22$ and $J^{m}=J^{f}=1.0, I^{m}=I^{f}=-0.3$, with external magnetic layer $H=0$. 
We can observe the beginning of the birth of skyrmions at the interface and in the interior magnetic layer.

Fig. 3 shows the ground state configuration for $J^{m f}=-1.22$ for first (surface) magnetic layer, with external magnetic layer $H=0.35$.

We can observe the skyrmions for the surface and interior magnetic layer. A zoom of a skyrmion shown in Fig. 3 and dependence the $z$-components of spins across the skyrmion of cites indicates that the skyrmion is of Bloch type (see Fig. 4).

Note that the skyrmions are found here in a range of sufficiently strong interface coupling and the applied field. The skyrmions are distributed in $3 \mathrm{D}$ space (not on a plane) in the magnetic layer and they are not uniform (see Fig. 3). In the case of a single magnetic layer skyrmions are uniform on a plane (2D sheet) (see [22]). And also as shown our calculations in a case where the frustration is sufficiently strong (see Fig. 5).

The GS configuration of the second (interior) magnetic layer also shows some texture with skyrmions. With increasing $\vec{H}$ we can observe skyrmions very pronounced for the surface layer but less contrast for the interior magnetic layer. For fields stronger than $H=0.43$, skyrmions disappear in interior layers. At strong fields, all spins are parallel to the field, thus no skyrmions anywhere.

Now we consider a case with the frustrated regime with $J^{m}=J^{f}=1.0,\left(I^{m}, I^{f}\right) \in(-0,45,0)$ above the critical value -0.5 , below this value, the antiferromagnetic ordering replaces the ferromagnetic ordering.

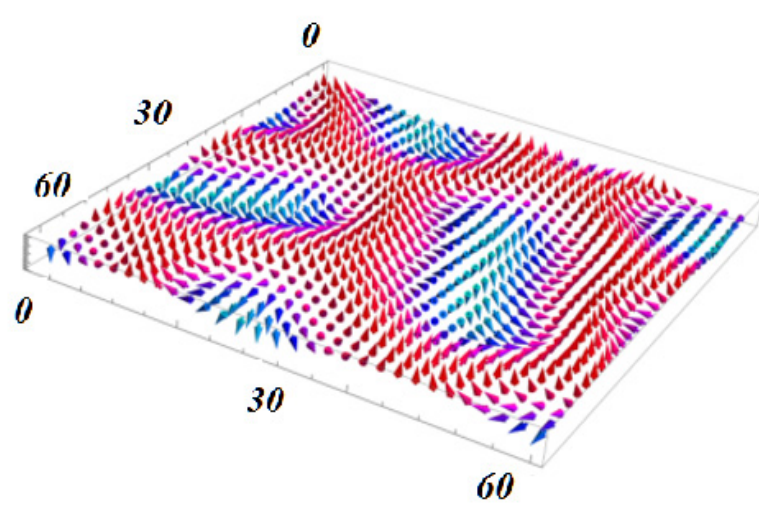

Fig. 2. (Color online) 3D view of the GS configuration of the surface magnetic layer for $J^{m f}=-1.22$ and $J^{m}=J^{f}=1.0, I^{m}=I^{f}=0$, with external magnetic layer $H=0.15$.

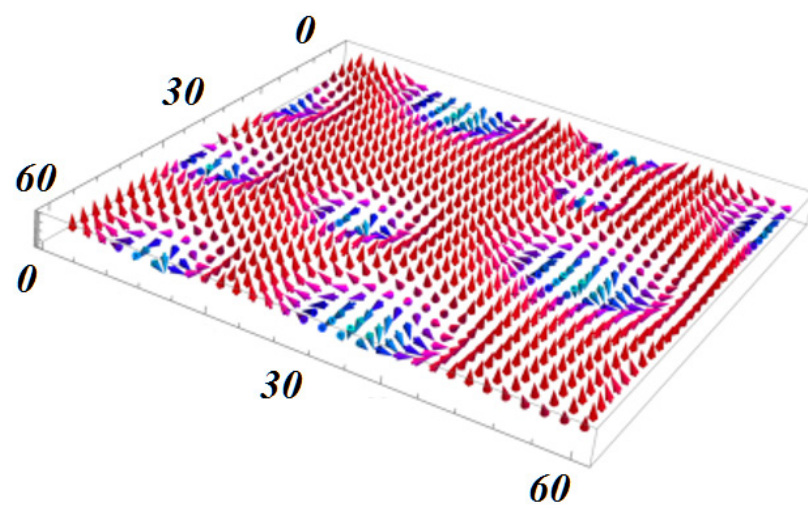

Fig. 3. (Color online) 3D view of the GS configuration of the surface magnetic layer for $J^{m f}=-1.22$ and $J^{m}=J^{f}=1.0, I^{m}=I^{f}=0$, with external magnetic layer $H=0.35$.
At this field strength $H=0.35$ if we increase the frustration, for example $I^{m}=I^{f}=-0.4$, then the skyrmion structure is enhanced: we can observe a clear $3 \mathrm{D}$ skyrmion crystal structure not only in the interface layer but also in the interior layers. This is shown in Fig. 5.

We can observe a clear 3D skyrmion crystal structure in the whole magnetic layers, not only near the interface layer. Unlike the case where we do not take into account the interaction between NNN, in the present case where the frustration is very strong we see that a large number of skyrmions are distributed over the whole magnetic layers with a certain periodicity close to a perfect crystal.

\section{Skyrmion phase transition}

The magnetic transition is driven by the competition between $T$, the DM interaction (namely $\left\langle P_{k}\right\rangle$ ), the field $\vec{H}$ and the magnetic texture (skyrmions). The stronger DM or $J^{m f}$, the higher the transition temperature $T_{C}$ of the skyrmion structure. As mentioned above strong DM interaction helps to stabilize the skyrmion crystal $[18,19]$ at the superlattice interface. We use a strong $J^{m f}$ as in the previous section.
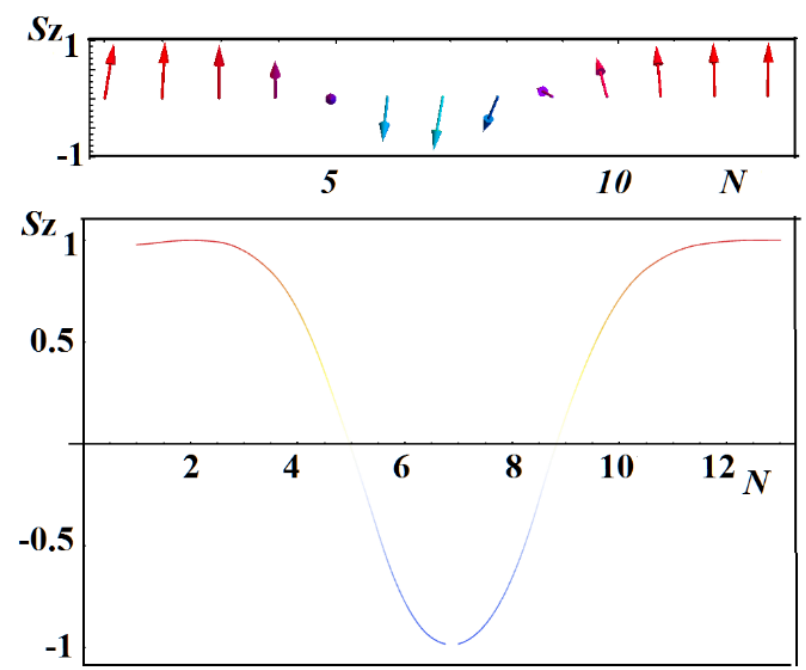

Fig. 4. (Color online) $\mathrm{z}$-components of spins across the skyrmion. Parameters: $J^{m f}=-1.22$ and $J^{m}=J^{f}=1.0, I^{m}=I^{f}=0$, with external magnetic layer $H=0.35$.

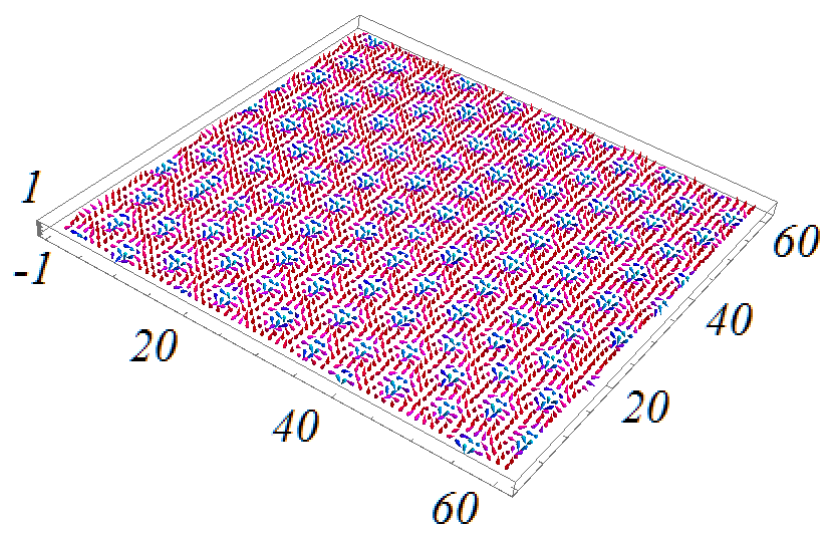

Fig. 5. (Color online) 3D view of the GS configuration of the surface magnetic layer for $J^{m f}=-1.22$ and $J^{m}=J^{f}=1.0, I^{m}=I^{f}=-0.4$, with external magnetic layer $H=0.35$ (the GS configuration of the interior magnetic layer have the similar texture). 
We use the Metropolis algorithm [20,21] to calculate physical quantities of the system at finite temperatures $T$. Simulation times are $10^{6}$ Monte Carlo steps (MCS) per spin for equilibrating the system and $10^{6} \mathrm{MCS} /$ spin for averaging. We calculate the internal energy and the layer order parameters of the magnetic $\left(O P_{m}\right)$ and ferroelectric $\left(O P_{f}\right)$ films.

For the ferroelectric layers, the order parameter $O P_{f}$ of layer $n$ is defined as the polarization

$$
O P_{f}(n)=\frac{1}{N^{2}}\left\langle\left|\sum_{i \in n} P_{i}^{z}\right|\right\rangle
$$

where $\langle\ldots\rangle$ denotes the time average.

For the magnetic layers we define the order parameter as the projection of an actual spin configuration at a given $T$ on its GS and we take the time average. This order parameter of layer $n$ is thus defined as

$$
O P_{m}(n)=\frac{1}{N^{2}\left(t_{a}-t_{0}\right)} \sum_{i \in n}\left|\sum_{t=t_{0}}^{t_{a}} \vec{S}_{i}(T, t) \cdot \vec{S}_{i}^{0}(T=0)\right|
$$

where $\vec{S}_{i}(T, t)$ is the $i$-th spin at the time $t$, at temperature $T$, and $\vec{S}_{i}^{0}(T=0)$ is its state in the GS. The order parameter $O P_{m}(n)$ is close to 1 at very low $T$ where each spin is only weakly deviated from its state in the GS. $O P_{m}(n)$ is zero when every spin strongly fluctuates in the paramagnetic state. In our case of skyrmion structure, the GS is stable up to a finite $T$.

Fig. 6 shows the magnetic order parameter versus $T$. The green and blue (Fig. 6 a) lines correspond to $O P_{m}$ for $J^{m f}=-1.22$ with $H^{z}=0.35$ and $H^{z}=0$, respectively; the blue and gold lines correspond to $O P$ for $J^{m f}=-6$ with $H^{z}=0$ and $H^{z}=1$. These curves indicate phase transitions at $T_{c}^{m}=0.72$ for $J^{m f}=-1.22$ with $H^{z}=0.35$ (green) and second order phase transition at $T_{c}^{m}=0.83$ for $J^{m f}=-1.22$ with $H^{z}=0.0$ (blue). Let us discuss about the nature of the transition in shown in Fig. 6 a. When $H \neq 0$, the first transition at low temperature $(T \cong 0.72-1.25)$ is due to the destruction of the scyrmion structure. After this transition, the $z$ spin components being not zero under an applied field come close to zero only at high ( $T \cong 2.3)$. This is not a phase transition because the $z$ components will never be zero in a field if $J^{m f}$ is not so strong. For large value of $J^{m f}$ the dependence order parameter of surface magnetic layers versus temperature indicate two phase transitions $\left(J^{m f}=-6.0, H^{z}=1\right)$ (blue). In the case of zero field, namely $\left(J^{m f}=-6.0, H^{z}=0\right)$ (gold), one has a first-order phase transitions occurring at $T_{c}=2.30$. When $J^{m f}$ is very strong $\left(J^{m f}=-6\right.$, blue data points $)$, the DM interaction is so strong that the spins will lie in the $x y$ plane in spite of $H$ : we see that the $z$ components are zero after the loss of the ferroelectric ordering at $T \cong 2.3$. Note that when $H=0$ (gold data points), there is no skyrmions, the spin configuration is chiral (helical). The single transition to the paramagnetic phase occurs at $T \cong 2.3$ where the chiral ordering and the ferroelectric ordering are lost at the same time (see Fig. 6b).

Fig. 7 shows the magnetic (purple) and ferroelectric (green) energies versus $T$ for $\left(J^{m f}=-6.0, H^{z}=0\right)$.

One sees the discontinuities of these curves at $T_{c}=2.30$ indicating the first-order transitions for both magnetic and ferroelectric at the same temperature. In fact, with such a strong $J^{m f}$ the transitions in both magnetic and ferroelectric films are driven by the interface, this explains the same $T_{c}$ for both.
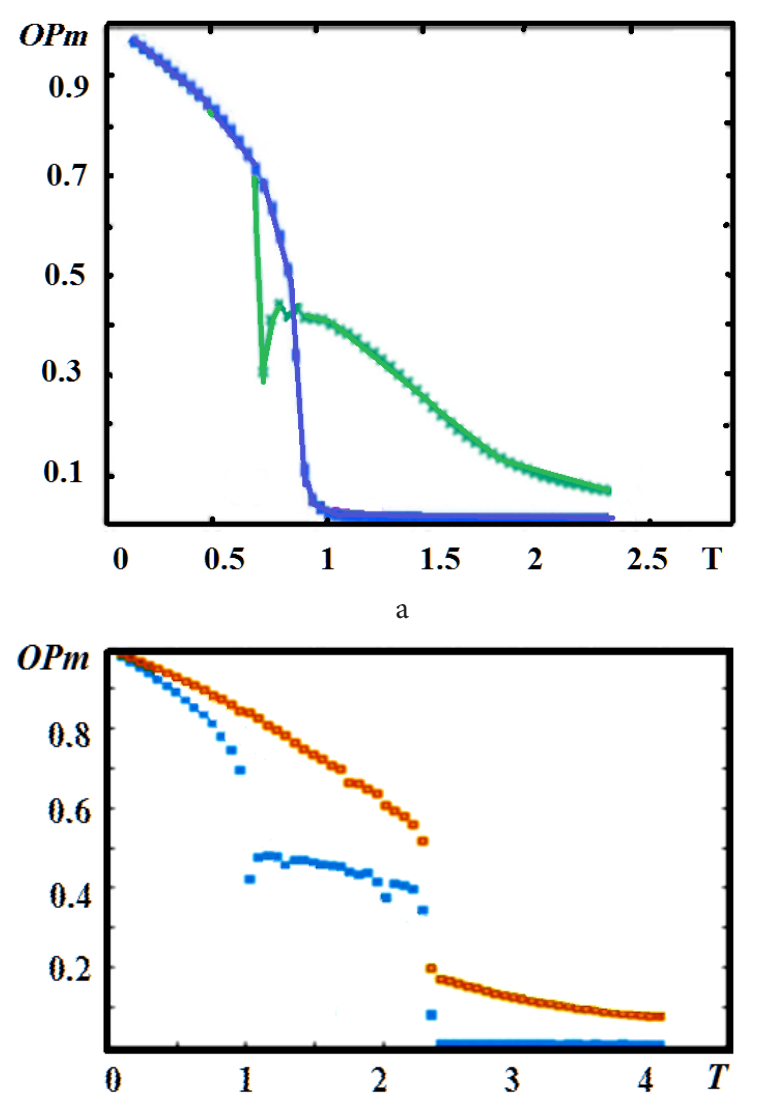

b

Fig. 6. (Color online) The green and blue lines correspond to $O P_{m}$ for $\left(J^{m f}=-1.22, H^{z}=0.35\right)$ and $\left(J^{m f}=-1.22, H^{z}=0.0\right), \quad J^{m}=J^{f}=1^{m}$, $I^{m}=I^{f}=0 \quad$ (a); Order parameter of magnetic film versus $T$. The blue and gold dots correspond to $O P_{m}$ for $\left(J^{m f}=-6.0, H^{z}=1.0\right)$ and $\left(J^{m f}=-6.0, H^{z}=0\right) . J^{m}=J^{f}=1, I^{m}=I^{f}=0(\mathrm{~b})$.

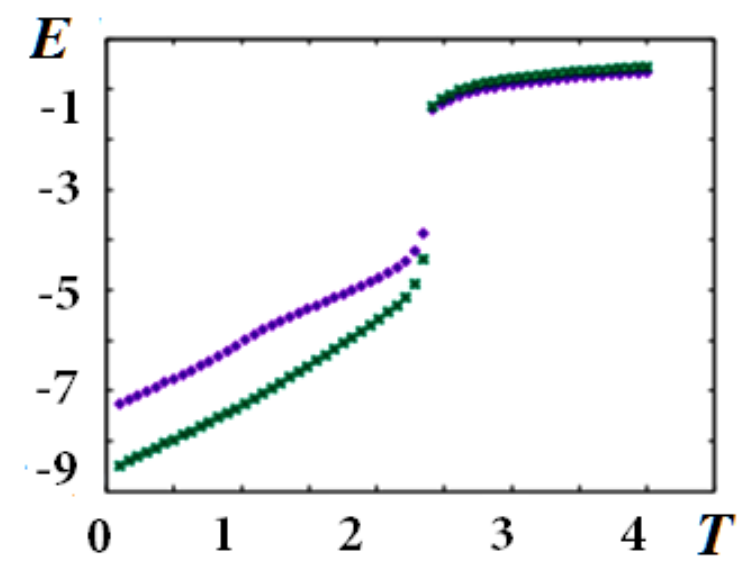

Fig. 7. (Color online) Energies of magnetic (purple dots) and ferroelectric (green dots) subsystems versus $T$ for $\left(J^{m f}=-6.0\right.$, $\left.J^{m}=J^{f}=1, I^{m}=I^{f}=0 \quad H^{z}=0\right)$.

\section{Conclusion}

In this article, we have studied a new model for the interface coupling between a ferromagnetic film and a ferroelectric film in a superlattice of multiferroic. This interaction has the form of a Dzyaloshinskii-Moriya (DM) interaction between the order parameters of ferroelectric films and the 
spins of ferromagnetic layers at the interface. We have taken into account the frustration due to the NNN interactions in both magnetic and ferroelectric layers. The ground state shows uniform non collinear spin configurations in zero field and skyrmions in an applied magnetic field. Monte Carlo simulation has been used to study the phase transition occurring in the superlattice with and without applied field. Skyrmions have been shown to be stable at finite temperatures. We have also shown that the nature of the phase transition can be of second or first order, depending on the value of magnetoelectric interaction. As expected, the magnetic frustration enhances creation of skyrmions. The existence of skyrmions confined at the ferromagnetic-ferroelectric interface is very interesting. We believe that it can be used in transport applications in spintronic devices.

Acknowledgements. The authors want to thank for support and discussions prof. Hung T. Diep (LPTM, University of Cergy Pontoise, France).

\section{References}

1. I. A. Sergienko, E. Dagotto. Physical Review B. 73 (9), 094434 (2006). $\underline{\text { Crossref }}$

2. A.P. Pyatakov. Physica B: Condensed Matter. 542, 59 (2018). Crossref

3. T. Maruyama, Y. Shiota, T. Nozaki, et al. Nature nanotechnology. 4 (3), 158 (2009). Crossref

4. O.G. Udalov, I.S. Beloborodov. AIP Advances. 8 (5), 055810 (2018). $\underline{\text { Crossref }}$

5. A. R. Yuldasheva, N. M. Nugaeva. Letters on Materials. 9 (3), 354 (2019). (in Russian) Crossref

6. A. Alberca, C. Munuera, et al.. Scientific reports. 5, 17926 (2015). $\underline{\text { Crossref }}$
7. H. Katsura, N. Nagaosa, A. V. Balatsky. Physical review letters. 95 (5), 057205 (2005). Crossref

8. S.-W. Cheong, M. Mostovoy. Nature materials. 6 (1), 13 (2007). Crossref

9. A. N. Bogdanov, D. Yablonskii. Sov. Phys. JETP. 68 (1), 101 (1989).

10. U. Rößler, A.N. Bogdanov, C. Pfleiderer. Nature. 442 (7104), 797 (2006). $\underline{\text { Crossref }}$

11. A. Yadav, C. Nelson, S. Hsu, et al. Nature. 530 (7589), 198 (2016). Crossref

12. A. Fert, V. Cros, J. Sampaio. Nature nanotechnology. 8 (3), 152 (2013). Crossref

13. R. Tomasello, E. Martinez, R. Zivieri, L. Torres, M. Carpentieri, G. Finocchio. Scientific reports. 4, 6784 (2014). Crossref

14. W. Koshibae, Y. Kaneko, J. Iwasaki, M. Kawasaki, Y. Tokura, N. Nagaosa. Japanese Journal of Applied Physics. 54 (5), 053001 (2015). $\underline{\text { Crossref }}$

15. W. Kang, Y. Huang, C. Zheng, W. Lv, N. Lei, Y. Zhang, X. Zhang, Y. Zhou, W. Zhao. Scientific reports. 6, 23164 (2016). $\underline{\text { Crossref }}$

16. I. F. Sharafullin, M. Kh. Kharrasov, H. T. Diep. Phys. Rev. B. 99, 214420 (2019). Crossref

17. S. El Hog, A. Bailly-Reyre, H.T. Diep. Journal of Magnetism and Magnetic Materials. 455, 32 (2018). Crossref

18. H. Yang, G. Chen, A.A. C. Cotta, A.T.N’Diaye, S. A. Nikolaev et al. Nat. Mater. 17, 605 (2018). Crossref

19. A. Manchon, H. C. Koo, J. Nitta, S. Frolov, R. Duine. Nat. Mater. 14, 871 (2015). Crossref

20. H. T. Diep. Theory of magnetism - Application to surface physics. World Scientific (2014) 420 p. $\underline{\text { Crossref }}$

21. A.K. Murtazaev, A. B. Babaev. Materials Letters. 238, 321 (2019). Crossref

22. H.T. Diep. Entropy. 21 (2), 175 (2019). Crossref 